\title{
Acción de flavonoides sobre la conversión de ácidos grasos poliinsaturados de cadena larga a partir de ácidos grasos esenciales
}

\section{Action of flavonoids on conversion of long chain polyunsaturated fatty acids from essential fatty acids}

\begin{abstract}
RESUMEN
Estudios epidemiológicos y clínicos han reportado múltiples beneficios a partir de la ingesta de ácidos grasos poliinsaturados de cadena larga n-3 (AGPI-CL n-3) EPA (ácido eicosapentaenoico) y DHA (ácido docosahexaenoico), sin embargo, la sobreexplotación de los recursos marinos limita su disponibilidad actual y futura. El ácido alfa-linolénico (ALA) es precursor metabólico de AGPI-CL n-3, por tanto su consumo es una alternativa a considerarse. No obstante, la conversión de ALA hacia EPA y DHA no es eficiente. Por otra parte, antioxidantes como los flavonoides incrementan la concentración sérica y tisular de AGPI-CL n-3, aunque los mecanismos subyacentes no están completamente dilucidados. Se explora la acción de los AGPI y flavonoides sobre el metabolismo de los AGPI-CLn-3, al modular factores de transcripción como los proliferadores de peroxisomas alfa $(P P A R-\alpha)$, la proteína de unión a los elementos regulatorios de esteroles (SREBP-1) y la expresión génica de las enzimas ácido graso desaturasas delta $5(\Delta 5)$ y delta $6(\Delta 6)$. También se recogen otras hipótesis que explicarían el incremento de AGPI n-3, como la acción antioxidante ejercida por los flavonoides y sus metabolitos.

Palabras clave: Antioxidantes; AGPIs; Desaturasas $\Delta 5$ y $\Delta 6$; Flavonoides; PPAR- $\alpha$, SREBP-1.
\end{abstract}

\footnotetext{
ABSTRACT

Epidemiological and clinical studies have reported multiple benefits from the intake of Long Chain Polyunsaturated Fatty Acids n-3 (LC-PUFA n-3), EPA (eicosapentaenoic acid) and $D H A$ (docosahexaenoic acid); however, overexploitation of marine sources limits current and future availability. The alpha-linolenic acid (ALA) is a metabolic precursor of PUFAs $n-3-L C$, therefore its consumption is an alternative to be considered. However, the conversion of ALA to EPA and DHA is not efficient. On other hand, antioxidants such as flavonoids increase serum and tissue concentration of PUFA LC n-3, although the underlying mechanisms are not fully elucidated. The effect of PUFAs and flavonoids on metabolism PUFAs n-3 LC. to modulate transcription factors such as peroxisome proliferators alpha receptor $(P P A R-\alpha)$, the sterol regulatory element-binding protein,
}

Sixto Reyna $G^{1,2}$, Rodrigo Valenzuela ${ }^{3}$, María Elena Villanueva ${ }^{1 *}$.

1. Universidad Nacional Agraria La Molina, Facultad de Zootecnia, Departamento de Nutrición. Lima - Perú

2. Universidad Técnica de Manabí. Carrera de Medicina Veterinaria, Departamento de Veterinaria. Manabi, Ecuador. 3. Universidad de Chile, Facultad de Medicina, Escuela de Nutrición y Dietética. Santiago. Chile.

\author{
Dirigir a correspondencia a: María Elena Villanueva. \\ Universidad Nacional Agraria La Molina, Facultad de Zootecnia, \\ Departamento de Nutrición. Av. La Molina s/n \\ E-mail: mvillanueva@lamolina.edu.pe
}

(SREBP 1) and genic expression of fatty acid desaturase enzymes delta $5(\Delta 5)$ and delta $6(\Delta 6)$ was explored. Other hypotheses that could explain the increase of $n-3$ PUFAs were also included such as antioxidant action, exerted by flavonoids and their metabolites.

Key words: Antioxidants; PUFAs, Desaturase $\triangle 5$ and $\Delta 6$; Flavonoids; PPAR- $\alpha$, SREBP-1.

\section{INTRODUCCIÓN}

A nivel global las enfermedades crónicas no transmisibles (ECNT) son la principal causa de discapacidad y mortalidad, así de los 57 millones de decesos en el año 2008, 36 millones (63\%) se atribuyeron a las ECNT. El $80 \%$ de estas muertes (29 millones) ocurrieron en países de ingresos medios o bajos. 
La manifestación de las ECNT y la consecuente pérdida de productividad, repercuten negativamente en la economía familiar y nacional, estimándose que anualmente, sólo los costos de servicios de salud empujan a la pobreza a 100 millones de personas, particularmente en las naciones más pobres'.

Las ECNT se asocian a factores dietarios, entre ellos elevado consumo de ácidos grasos saturados, desequilibrio en la ingesta de ácido linoleico (C18:2 n-6, AL) y ácido $\alpha$-linolénico (C18:3 n-6, ALA) -precursores de los AGPI CL $\mathrm{n}-6$ y $\mathrm{n}-3$, respectivamente- $\mathrm{e}$ insuficiente consumo de ácido eicosapentaenoico (C20:5 n-3, EPA) y docosahexaenoico $(\mathrm{C} 22: 6, \mathrm{DHA})^{2}$; la dieta occidental presenta una relación de 10: 1 a 20:1, e incluso 30:1 respecto a la ingesta de AGPI $\mathrm{n}-6: \mathrm{n}-3$, superior a la tasa recomendada de $4: 1^{3}$.

Los AGPI n-3 son componentes fundamentales de los fosfolípidos de las membranas celulares, reflejando la composición de la dieta ${ }^{4}$, ejerciendo efectos biológicos sobre el metabolismo de lípidos mediante la síntesis y acción de eicosanoides ${ }^{4}$, e incrementando, en ratones inducidos a esteatosis hepática, la abundancia nuclear de los receptores activados por proliferadores de peroxisomas alfa, PPAR- $\alpha$ del inglés "peroxisome proliferator-activated receptor- $\alpha$ " (PPAR- $\alpha)$, y reduciendo la cuantía nuclear de la proteína de unión a los elementos regulatorios de esteroles 1c, SREBP-1c, por sus siglas en inglés "Sterol Regulatory Element-Binding Proteins", lo que incrementa la $\beta$ - oxidación y disminuye la lipogénesis ${ }^{5}$, por tanto los AGPI-CL tienen un papel clave en la prevención y manejo de las ECNT ${ }^{4}$.

Adicionalmente, la incorporación de DHA en los fosfolípidos del tejido nervioso de humanos y modelos animales durante la gestación y la lactancia se asocia con el desarrollo del sistema nervioso y la función visual ${ }^{6,7}$, y su incorporación depende principalmente de la transferencia a través de la placenta y leche materna, respectivamante ${ }^{6}$.

La provisión de EPA y DHA a través de la explotación de recursos marinos, no es sostenible ${ }^{8}$, en este contexto, se ha propuesto la obtención de estos ácidos grasos a partir de ALA, considerando que es precursor de EPA y DHA ${ }^{9}$, sin embargo la eficiencia de este proceso es limitada: en humanos la tasa de conversión de ALA hacia EPA es menor al 8\%, mientras la conversión de ALA hacia DHA es inferior a $4 \%{ }^{10}$.

Ciertos antioxidantes interactúan con el metabolismo lipídico, así el consumo de flavonoides como las antocianinas aumenta la actividad y expresión génica de PPAR- $\alpha$ en ratas obesas ${ }^{11}$ y disminuye la expresión de SREBP $1 c^{12}$. La ingesta de flavonoides también incrementa los niveles plasmáticos de EPA y DHA, aunque no está claro el mecanismo subyacente a este efecto ${ }^{13}$, por tanto la presente revisión hace referencia a las principales fuentes de ALA, la conversión de los AGPI-CL a partir de sus precursores (AL y ALA), la acción y regulación de las enzimas desaturasas, el papel de factores de transcripción (PPAR- $\alpha$ y SREBP-1) y probables mecanismos de acción de los antioxidantes en este proceso.

\section{MATERIALES Y MÉTODOS}

La revisión incluyó información referente al metabolismo de estos ácidos grasos con énfasis en la asociación entre la ingesta de polifenoles como los flavonoides y los niveles de AGPI-CL en humanos y animales, por ello se realizó una búsqueda bibliográfica utilizando la base de datos PubMed de the National Library of Medicine-National Institutes of Health, utilizando los términos "increased EPA and DHA" junto con "anthocyanins", "flavonoids", "polyphenols" y "wine"; además de "purple corn and PPAR SREBP expression", wine consumption polyunsaturated lipids", polyphenols and lipid peroxidation", polyphenols and lipid profile in metabolic síndrome y polyphenols and fatty acids n-3 in plasma".

\section{Conversión de AL y ALA hacia AGPI-CL}

El AL y ALA contienen el primer doble enlace cis entre el sexto y el séptimo átomo de carbono (n-6) o el tercer y cuarto átomo de carbono (n-3) a partir del grupo metilo final de los ácidos grasos ${ }^{14}$. AL y ALA son considerados esenciales y deben obtenerse a partir de la dieta ${ }^{15}$ ya que las células animales carecen de enzimas desaturasas capaces de añadir enlaces dobles a los ácidos grasos en los últimos nueve carbonos de la molécula, lo que impide la síntesis de novo de AGPI ${ }^{13,16}$.

La síntesis de AGPI-CL n-3 y n-6 a partir de ALA y AL es posible por una secuencia de desaturaciones y elongaciones catalizadas en ambos casos, por las enzimas desaturasas $\Delta 5$ y $\Delta 6(\Delta 5 \mathrm{D}$ y $\Delta 6 \mathrm{D})$ y presumiblemente elongasas 2 y $5^{17}$. En consecuencia, la conversión de ALA hacia EPA compite con la conversión de $\mathrm{AL}$ hacia el $\mathrm{AA}^{16,18}$.

Por acción de $\triangle 6 \mathrm{D}, \mathrm{ALA}$ es convertido a ácido estearidónico (18:4n-3), que es elongado hacia ácido eicosatetraenoico (20:4n-3). Una posterior desaturación, catalizada por $\Delta 5 \mathrm{D}$, conduce a la síntesis de $\mathrm{EPA}^{18}$, que por acción de elongasa-2 genera 22:5n-3; $\Delta 6 \mathrm{D}$ cataliza la conversión de 24:5n-3 a 24:6n-3. Estas reacciones ocurren en el retículo endoplásmico del hepatocito; posteriormente 24:6n-3 es transferido a los peroxisomas de las células hepáticas, donde es sometido a $\beta$-oxidación para formar DHA $(22: 6 n-3)^{10}$. En los peroxisomas también se produce la retro-conversión de $\mathrm{DHA}$ hacia $\mathrm{EPA}^{19}$, este proceso no es muy eficiente en humanos ${ }^{20}$, particularmente en células neuronales ${ }^{19}$.

Probablemente la conversión de EPA hacia DHA es menor que de ALA hacia EPA debido a que implican reacciones adicionales de elongación, desaturación y $\beta$-oxidación, que están sujetas a regulación hormonal ${ }^{21}$. Así el nivel plasmático de AGPI-CL, en animales y humanos, es superior en hembras que en machos, fenómeno atribuido a los estrógenos, que median la regulación al alza la expresión de los genes de desaturasas $\Delta 5 \mathrm{D}$ y $\Delta 6 \mathrm{D}^{10,22}$.

El AL es metabolizado a ácidos grasos n- 6 de cadena larga y con más insaturaciones, producto de la inserción de dobles enlaces adicionales mediante consecutivas elongaciones y desaturaciones ${ }^{3}$. El primer metabolito de 
la vía n-6 en mamíferos es el ácido $\gamma$ - linolenico (18:3n-6, GLA), por acción de la enzima $\Delta 6 \mathrm{D}^{3,14}$.

Posteriormente elongasa- 5 cataliza la conversión de GLA en ácido dihomo $\gamma$-linolénico (DGLA), que mediante desaturación catalizada por $\Delta 5 \mathrm{D}$ genera $\mathrm{ARA}^{3}$. En mamíferos, dos ciclos de elongaciones y una desaturación catalizada por $\Delta 6 \mathrm{D}$ sintetizan el ácido tetracosapentaenoico (24:5n-6), que es transferido del retículo endoplásmico a los peroxisomas, donde la $\beta$-oxidación genera el ácido docosapentaenoico $(22: 5 n-6)^{20}$. La síntesis de AGPI-CL n-6 y n-3 se aprecia en la figura 1.

Los productos principales de las vías $\mathrm{n}-6$ y $\mathrm{n}-3$ son el AA y DHA respectivamente, con EPA como un importante intermediario de la vía n-3 ${ }^{14}$. AA y DHA son los mayores componentes acilos de lípidos estructurales hallados en la mayoría de tejidos ${ }^{14}$.

A partir de AA y EPA, las enzimas lipooxigenasa (LO) y ciclo-oxigenasa (COX), catalizan la síntesis de eicosanoides ${ }^{20}$. Los eicosaniodes derivados de AA: prostaglandina E2, tromboxano B2 y leucotrieno B4, prostaciclinas y lipoxinas4 son proinflamatorios y más potentes que la prostaglandina E3, tromboxano B3 y leucotrieno derivados de EPA ${ }^{4}$.

El ácido dihomogammalinolénico (DGLA), precursor directo de AA en la vía n- 6 compite con AA y con EPA por LOX y COX. La acción de LOX/COX sobre DGLA genera las prostaglandinas y tromboxanos serie-1, con potentes efectos antiinflamatorios, vasodilatarores y antiagregantes ${ }^{14}$. Además de los eicosanoides, el metabolismo de EPA y DHA generan moléculas con efecto anti-inflamatorio, como las resolvinas de la serie $\mathrm{E}$ y $\mathrm{D}$, respectivamente. Adicionalmente, el metabolismo de $\mathrm{DHA}$ produce protectinas como docosatrienos y maresinas, que también ejercen actividad antiinflamatoria ${ }^{14}$.

\section{Enzimas desaturasas}

Delta 5 desaturasa $(\Delta 5 \mathrm{D})$ y delta 6 desaturasa $(\Delta 6 \mathrm{D})$ son enzimas clave en la conversión de AGPI n-6 y n-3, y son codificadas por los genes FADS1 y FADS2, respectivamente ${ }^{22}$. El hígado es el sitio primario para la síntesis de AGPI, ya que presenta la mayor actividad enzimática de $\triangle 6 \mathrm{D}$ y $\Delta 5 \mathrm{D}^{23}$, que son reguladas coordinadamente por el estatus nutricional, hormonal e inhibidas por retroalimentación por los productos finales (ARA, EPA y DHA), creando un control para su síntesis ${ }^{18}$.

\section{Factores de transcripción: SREBP Y PPAR}

Los factores de transcripción SREPB y PPARs juegan papeles clave en la regulación de $\triangle 5 \mathrm{D}$ y $\triangle 6 \mathrm{D}$ por $\mathrm{AGPI}^{24}$. Las proteínas SREBP pertenecen a la familia de los factores de transcripción del tipo básico hélice-bucle-hélice-cremallera de leucina (bHLH-Zip) ${ }^{25}$; las tres isoformas de SREBP, SREBP-1a y SREBP-1c y SREBP2 desempeñan diferentes roles en el metabolismo de lípidos ${ }^{26}$.

La expresión de SREBP-1a es alta en líneas celulares, mientras SREBP-1c lo es en células diferenciadas, incluyendo hepatocitos ${ }^{27}$; SREBP-1a activa la síntesis de ácidos grasos y colesterol, SREBP 1-c, que es el subtipo predominante en el hígado, estimula la síntesis de ácidos grasos y SREBP-2, la síntesis de colesterol ${ }^{28}$.

SREBP-2 se expresa de forma ubicua ${ }^{29} y$ es una de las isoformas predominantes en el hígado, donde preferentemente regula los genes involucrados en la biosíntesis de esteroles ${ }^{30}$ y presenta una moderada inducción de genes involucrados en la síntesis de ácidos grasos ${ }^{31}$.

Los PPARs son miembros de la súper-familia de receptores nucleares de ligandos activados por factores que regulan la expresión de genes específicos mediante mecanismos

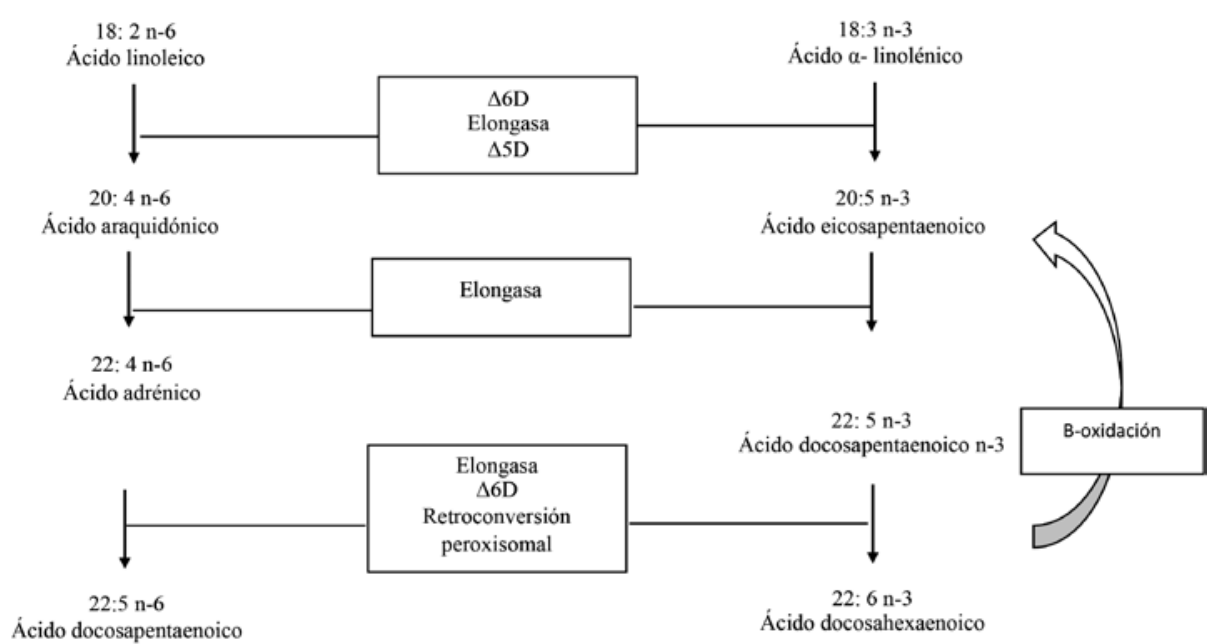

Figura 1. Metabolismo de las dos series de ácidos grasos poliinsaturados (AGPI). El ácido araquidónico es el principal LC-PUFA n-6 derivado del ácido linoleico. Los ácidos grasos eicosapentaenoico y docosahexaenoico son los dos principales LC-PUFA n-3 derivados del ácido $\alpha$-linolénico. Los LC-PUFA son sintetizados por sucesivas elongaciones y desaturaciones. La retro conversión peroxisomal determina la síntesis de EPA a partir de DHA79. 
comunes, se conocen tres isoformas de PPARs, $\alpha$ (alfa), $\beta / \delta$ (beta/delta) y $\gamma(\text { gamma })^{32}$.

PPAR- $\gamma$ es expresado principalmente en el tejido adiposo y sistema inmune ${ }^{25}$ y en músculo e hígado ${ }^{33}$; participa en la diferenciación de adipocitos y la sensibilidad a la insulina ${ }^{34}$. PPAR- $\beta / \delta$ se expresa de forma ubicua ${ }^{25}$, inhibe la producción de glucosa en el hígado e incrementa la $\beta$-oxidación y la termogénesis en el músculo cardiaco; reduce la respuesta inflamatoria de macrófagos y aumenta los niveles de $\mathrm{HDL}^{33}$. PPAR- $\alpha$ es expresado en el tejido adiposo marrón, hígado, músculo esquelético e intestinos delgado y grueso ${ }^{35}$.

Los PPARs se activan por concentraciones micromolares de AGPI-CL, estos se unen con mayor afinidad a PPAR- $\alpha$. El PPAR- $\alpha$ activado se une a los elementos de respuesta para PPAR, (PPRE, del inglés PPAR response element) que se encuentran en genes involucrados en el transporte $y$

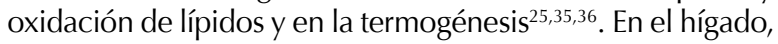
además de inducir la expresión de enzimas involucradas en la $\beta$-oxidación, PPAR- $\alpha$ incrementa la expresión de $\Delta 5 \mathrm{D}$ y $\Delta 6 \mathrm{D}^{23}$.

\section{Acción de SREBP en la regulación las desaturasas}

Los AGPI regulan la lipogénesis a través de SREBP-1. En ratas, estos ácidos grasos reducen la forma nuclear activa de SREBP-1, disminuyen la escisión de la proteína precursora de SREBP-1 y suprimen la expresión de su ARNm ${ }^{36,37}$. Ya que $\Delta 5 \mathrm{D}$ y $\Delta 6 \mathrm{D}$ son dianas de SREBP-1c, es probable que la disminución de la expresión génica de estas enzimas esté mediada por la supresión de SREBP 1-c ${ }^{23}$.

En contraste, una dieta baja en AGPI estimula la expresión de desaturasas y elongasas ${ }^{44}$. En el hígado, SREBP-1c activa los genes para la síntesis de ácidos grasos, incluyendo los de $\Delta 5 \mathrm{D}$ y $\Delta 6 \mathrm{D}^{23}$, ya que la región promotora del gen $\Delta 6 \mathrm{D}$ contiene un elemento regulatorio de esteroles de unión a SREBP-1 (SRE, por sus siglas en inglés sterol regulatory elements) ${ }^{37}$.

La sobreexpresión de SREBP-1c induce la expresión génica de $\Delta 6 \mathrm{D}$ y $\Delta 5 \mathrm{D}$ en el hígado de ratones transgénicos ${ }^{28}$. SRE también es requerido para la activación génica de $\Delta 6 \mathrm{D}$ humana por medio de SREBP-1 $\mathrm{C}^{27}$ y para la supresión génica de $\triangle 6 \mathrm{D}$ por acción de los $\mathrm{AGPI}^{39}$. Así pues, las enzimas desaturasas son cruciales para la producción de AGPI, que a su vez suprimen a SREBP- $1 \mathrm{c}^{23}$.

Los altos niveles de expresión de $\Delta 5 \mathrm{D}$ y $\Delta 6 \mathrm{D}$ en la glándula mamaria en ratones hembras lactantes sugieren la importancia de este tejido en la síntesis de AGPI presentes en la leche materna ${ }^{40}$. En ratas madres lactantes, la suplementación con aceite de chía como fuente de ALA no modificó la expresión génica de SREBP-1 en este tejido; dicha expresión se incrementó al adicionar un extracto de maíz morado como fuente de flavonoides ${ }^{41}$.

\section{Acción de PPAR- $\alpha$ en la regulación de las desaturasas}

Los PPARs pueden inducir a las desaturasas por mecanismos indirectos: incremento de la degradación de los ácidos grasos insaturados mediante el estímulo de enzimas involucradas en la $\beta$-oxidación a nivel mitocondrial y peroxisómico; la acción sobre los peroxisomas se traduce en mayores requerimientos de ácidos grasos insaturados por los fosfolípidos de membrana, resultando en inducción de las desaturasas. PPAR- $\alpha$ también actúa directamente sobre la inducción génica de $\Delta 6 \mathrm{D}$ al regular la síntesis de AGPI$\mathrm{CL}$ cuando existen bajos niveles de estos ácidos grasos ${ }^{36}$.

La activación de PPAR- $\alpha$ reduce la expresión de SREBP-1c en el hígado de ratas ${ }^{42}$. La aparente implicación de PPAR- $\alpha$ como un regulador positivo de la expresión génica de la $\triangle 6 \mathrm{D}$ presenta un conflicto mecanístico porque los AGPI dietarios, ligandos activadores de PPAR, reducen la abundancia de $A R N m$ y la actividad de la $\triangle 6 \mathrm{D}$ hepática ${ }^{43}$, no habiendo claridad sobre el mismo, ya que aunque los fibratos, activadores de PPAR- $\alpha$, regulan al alza la actividad y expresión de $\triangle 6 \mathrm{D}$, también se ha reportado que disminuyen los niveles de EPA y $\mathrm{DHA}^{13}$, esta aparente contradicción se explicaría por la acción de agonistas de PPAR- $\alpha$ en diferentes especies, así el ácido fenofíbrico y el ácido fenil acético incrementan la expresión de PPAR- $\alpha$ en hepatocitos de ratas, no así en las células hepáticas de humanos ${ }^{14}$.

\section{Regulación de AGPI en función a la afinidad enzimática por el sustrato}

La cantidad absoluta de ALA y el ratio AL: ALA influencia en la conversión de ALA a EPA debido a la competencia entre estos ácidos grasos por $\Delta 6 \mathrm{D}^{44}$, la enzima limitante en esta vía metabólica ${ }^{18}$, en este sentido se ha señalado que la síntesis de AGPI-CL n-3 depende más de la interacción competitiva de las enzimas relacionadas con la elongación y desaturación por el sustrato que de su expresión génica ${ }^{38}$. Asimismo, la elongación y desaturación de los ácidos grasos está sujeta a regulación por retroalimentación, ya que ARA y DHA suprimen la conversión endógena de $\mathrm{AL}$ y $\mathrm{ALA}$ en ácidos grasos más largos ${ }^{16}$.

Un estudio realizado en cerdos jóvenes determinó que el consumo de $\mathrm{AL}$ y $\mathrm{ALA}$ tiene un efecto adverso sobre la síntesis de AGPI-CL n-3 y n-6, respectivamente. Sin embargo, una elevada ingesta de ALA disminuye la concentración hepática de $\mathrm{DHA}$ e incrementa los niveles de $\mathrm{AL}^{17}$. La reducción de la síntesis de DHA se explicaría por la mayor afinidad de $\triangle 6 \mathrm{D}$ por ALA que por C24:5 n-3, lo cual inhibiría la desaturación de este ácido graso cuando existe abundancia de ALA, previniendo la síntesis de $\mathrm{DHA} \mathrm{A}^{17}$. El aumento de $\mathrm{AL}$ se atribuye a la mayor tasa de oxidación de ALA, que fluctúa entre $60-85 \%$, mientras es de $65 \%$ para DHA, $30 \%$ en caso de ARA y $50 \%$ para AGPI n- $6^{16}$.

Se ha señalado que es posible incrementar el nivel de $\mathrm{DHA}$ en ratas alimentadas con dietas que contengan ALA como única fuente de AGPI n-3, cuando su concentración sea inferior a 3\% de la energía dietaria. Así, el incremento de DHA no dependería únicamente de un mayor contenido de ALA en la dieta, lo que explicaría aparentes contradicciones entre diversos estudios ${ }^{45}$. Por otra parte, la acumulación de ALA y su conversión en ácidos grasos de cadenas más largas es un proceso tejido dependiente ${ }^{16}$. 


\section{Flavonoides y metabolismo de AGPI-CL}

Una vez que "la paradoja francesa" evidenció que el consumo de bebidas alcohólicas se asociaba con menor incidencia de enfermedades cardio y cerebro vasculares ${ }^{46}$, se atribuyó, al consumo de bebidas alcohólicas en humanos ${ }^{47}$, y etanol en ratas $^{48}$, un efecto protector, mediado por el incremento de AGPI CL en diversos tejidos, postulándose que el etanol podía modificar la actividad enzimática de $\Delta 5$ y $\Delta 6 \mathrm{D}$, incidiendo en la síntesis de AGPI-CL ${ }^{49}$. Posteriormente, estudios epidemiológicos sugirieron que la ingesta de flavonoides incrementaba la conversión de AGPI n-3 EPA y DHA a partir de $\mathrm{ALA}^{61}$. Varios de estos estudios en humanos, animales y cultivos celulares se describen en la tabla 1.

En individuos sanos o con dolencias cardiacas, el incremento de la concentración de AGPI n-3 se debería a compuestos no etanólicos presentes de modo particular en el vino ${ }^{50,51}$, planteándose que los polifenoles presentes en el mismo estimulan la biosíntesis de estos ácidos grasos, al activar reacciones de elongación y desaturación ${ }^{50}$. También en sujetos sanos, se atribuyó a la capacidad antioxidante de los polifenoles la inhibición de la peroxidación de los AGP ${ }^{50,52}$.

El suministro de un maíz genéticamente modificado, rico en flavonoides, en especial de antocianinas, incrementó la concentración plasmática de EPA y DHA en ratas ${ }^{13}$. Similares resultados se obtuvieron al suministrar, también a ratas macho, un jugo de arándano rico en antocianinas ${ }^{53}$, sugiriéndose que el incremento de AGPI se debía a la acción de las antocianinas sobre las $\Delta 5$ y $\Delta 6 \mathrm{D}$ en acción mediada por PPAR- $\alpha$ en ratas ${ }^{13}$ como en humanos $^{49}$, sin embargo estos estudios no evaluaron la influencia de las antocianinas sobre la actividad de las desaturasas.
En contraste a estudios previos que evaluaron la acción de alimentos ricos en antocianinas sobre el metabolismo de los $\mathrm{AGP}^{13,53}$, el suministro de antocinianinas no influyó en el perfil lipídico y la expresión génica de FADS2 (gen de la desaturasa 2, o $\triangle 6 \mathrm{D}$ ) en ratas ${ }^{54}$, estas divergencias probablemente se expliquen en función el perfil de antocianinas empleado por Vauzour et al, rico en delfinina 3 glucósico ${ }^{54}$, mientras que en trabajos previos la antocianina predominante era la cianidina 3 glucósido ${ }^{13,53}$.

Así mismo Vauzour et al. ${ }^{54}$, no encontraron variaciones en el perfil lipídico en el plasma de mujeres post menopáusicas al suministrar un extracto de sauco rico en antocianinas, no obstante, en este grupo etario el nivel de estrógenos es menor que en mujeres en edad fértil. Al respecto existe evidencia que la síntesis de AGPI n-3 está ligada al estrógeno ${ }^{55}$, ello explicaría por qué la concentración de AGPI n-3 es más alta en mujeres que en hombres.

A nivel hepático las antocianinas activan a la protein quinasa A activada por AMP (AMPK por sus siglas en inglés AMP- activated protein kinase) y esta a su vez incrementa la expresión génica de PPAR- $\alpha^{12,56}$. Se postula que la fosforilación y la actividad transcripcional de PPAR- $\alpha$ se incrementa mediante la activación de varios sistemas de protein kinasa, que son activados por receptores de estrógenos ligados a la membrana ${ }^{57}$, estimulando la síntesis de AGPI-CL mediante la inducción de desaturasas y elongasas ${ }^{58}$.

En contraste, AMPK reduce la expresión génica de SREBP-1 y de sus genes diana, entre ellos ACC y ácido graso sintasa (FAS), por tanto la activación de AMPK hepática incrementaría la $\beta$-oxidación y simultáneamente reduciría la lipogénesis ${ }^{59,56,13}$. En la figura 2 se resume la interacción de las antocianinas con el metabolismo de los AGPI.

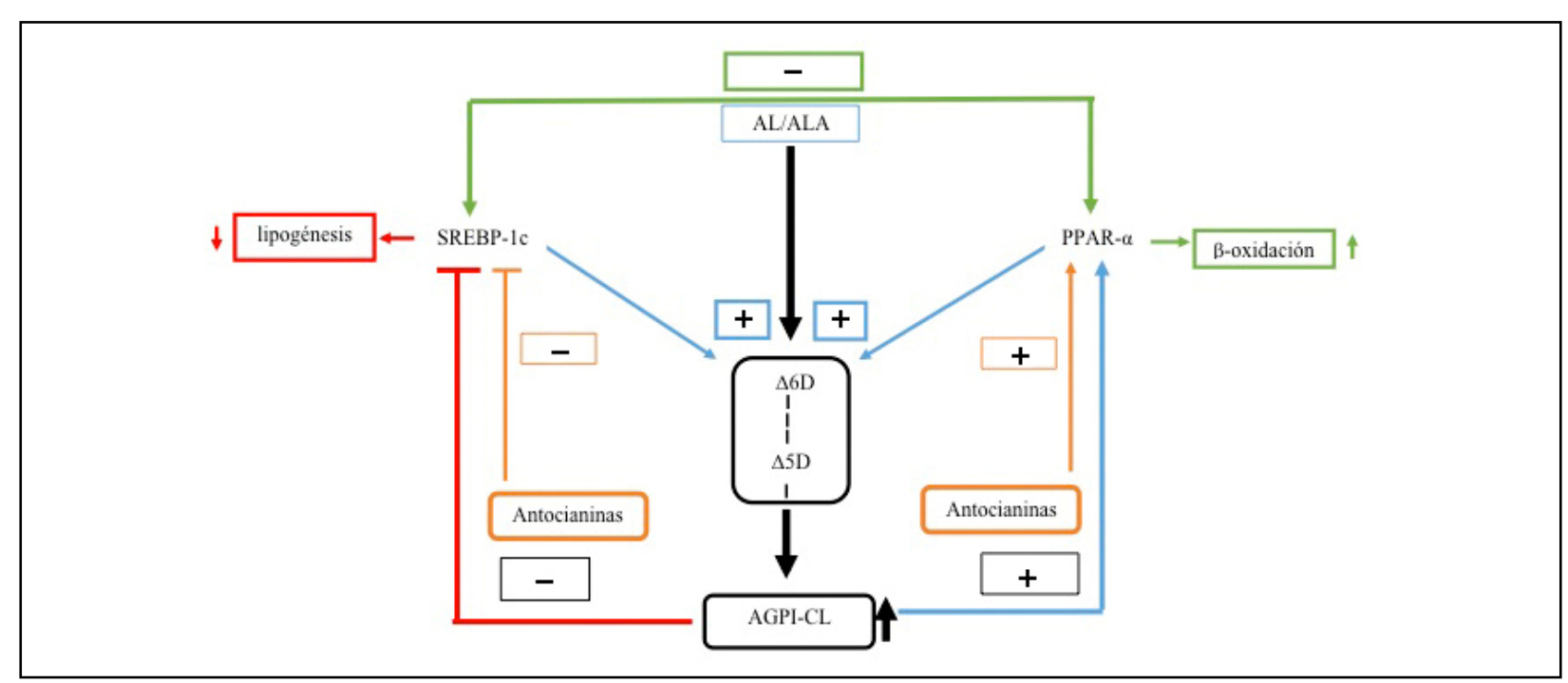

Figura 2. Regulación de las antocianinas sobre el metabolismo lipídico hepático al mediar sobre PPAR- $\alpha$ y SREBP-1 y estos sobre $\Delta 5 \mathrm{D}$ y $\triangle 6 \mathrm{D}$. AGPI-CL actúan como ligandos de PPAR- $\alpha$, estimulando la síntesis de AGPI-CL y la oxidación de ácidos grasos. Simultáneamente AGPI-CL disminuye la expresión de SREBP-1, reduciendo la síntesis de AGPI-CL y lipogénesis. Las antocianinas y PUFA ejercen similar efecto sobre PPAR- $\alpha$ y SREBP-1. PPAR- $\alpha$ reduce la expresión génica de SREBP-1. AL, ácido linoleico; ALA, ácido alfa-linolénico; $\Delta 6 \mathrm{D}$, delta 6 desaturasa; $\triangle 5 \mathrm{D}$, delta 5 desaturasa; LC-PUFA, ácidos grasos poliinsaturados de cadena larga; SREBP-1 proteína 1 de unión a los elementos reguladores de esteroles; PPAR- $\alpha$, receptor alfa activados por proliferadores de peroxisomas. 


\begin{tabular}{|c|c|c|c|c|c|c|}
\hline \begin{tabular}{|l|} 
Modelo \\
experimental \\
(Estudios clínicos \\
o modelos animales)
\end{tabular} & Duración & $\begin{array}{l}\text { Fuente de } \\
\text { polifenoles y } \\
\text { ácidos grasos }\end{array}$ & Dosis/concentración & Efecto & $\begin{array}{l}\begin{array}{l}\text { Mecanismo de acción } \\
\text { propuesto }\end{array} \\
\end{array}$ & Referencia \\
\hline $\begin{array}{l}36 \text { ratas macho } \\
\text { Wistar }(75-100 \mathrm{~g})\end{array}$ & 12 semanas & Trigo integral (WRy) & $\begin{array}{l}39 \% \text { y } 79 \% \text { de dietas } \\
\text { experimentales } \\
\text { corresponden a WR }\end{array}$ & $\begin{array}{l}\uparrow A G P I C L \text { n-3 en } \\
\text { sangre e hígado } \\
\text { Mejora de } \\
\text { composición } \\
\text { microbiota intestinal }\end{array}$ & $\begin{array}{l}\text { Síntesis de AGPI CL, } \\
\text { mediada por metabolitos } \\
\text { hepáticos y microbianos de } \\
\text { polifenoles. Posible efecto } \\
\text { del ácido fenólico. }\end{array}$ & $\begin{array}{l}\text { Ounnas et } \\
\text { al., } 2017^{67}\end{array}$ \\
\hline $\begin{array}{l}24 \text { Ratas macho } \\
\text { ( } 24 \text { meses de edad) }\end{array}$ & 4 semanas & $\begin{array}{l}\text { Naringenina (NA) } \\
\text { Hesperetina (HES) }\end{array}$ & $\begin{array}{l}\text { Administración oral } \\
\text { de } 15 \mathrm{mg} / \mathrm{kg} / \mathrm{d} \text { ía de } \\
\text { NA o HES. }\end{array}$ & $\begin{array}{l}\uparrow A G P I n-3 \text { y n-6. } \\
\downarrow \text { Proporción n-6/n-3 } \\
\uparrow \text { Actividad de SOD1, } \\
\text { SOD2, GPx, GR y } \\
\text { GSH en el hígado }\end{array}$ & $\begin{array}{l}\text { Probable acción de } \\
\text { flavonoides sobre } \Delta 5 \text { y } \\
\Delta 6 \mathrm{D} \text { y control del daño } \\
\text { oxidativo }\end{array}$ & $\begin{array}{l}\text { Miler et al., } \\
2016^{64}\end{array}$ \\
\hline $\begin{array}{l}24 \text { ratas macho } \\
\text { Wistar }(75-100 \mathrm{~g})\end{array}$ & 12 semanas & $\begin{array}{l}\text { Centeno integral } \\
\text { (WR) y centeno } \\
\text { refinado }(R R)\end{array}$ & $\begin{array}{l}\text { Dietas experimentales } \\
\text { preparadas con } 50 \% \\
\text { de WW o RR. }\end{array}$ & $\begin{array}{l}\text { WR } \uparrow \text { EPA y DHA } \\
\text { plasmático y hepático } \\
\text { y la diversidad } \\
\text { bacteriana en heces, } \\
\text { cambios marginales } \\
\text { de microbiota cecal }\end{array}$ & $\begin{array}{l}\text { WR estimula la síntesis } \\
\text { endógena de AGPI-CL n-3 } \\
\text { Polifenoles protege a } \\
\text { AGPI-CL de oxidación }\end{array}$ & $\begin{array}{l}\text { Ounnas et } \\
\text { al., } 2016^{66}\end{array}$ \\
\hline $\begin{array}{l}\text { Exp. 1: } 40 \text { Ratas } \\
\text { macho, línea Wistar } \\
7-8 \text { semanas }\end{array}$ & $\begin{array}{l}\text { Exp. 1: } 8 \\
\text { semanas }\end{array}$ & $\begin{array}{l}\text { Expr. 1: Antocianinas } \\
\text { pura }\end{array}$ & $\begin{array}{l}\text { Exp. 1: } 240 \mathrm{mg} / \mathrm{kg} \\
\text { dieta (aprox. } \\
11 \mathrm{mg} / \mathrm{kg} / \text { día/rata). }\end{array}$ & $\begin{array}{l}\text { No modificación } \\
\text { del perfil lipídico los } \\
\text { modelos empleados } \\
\text { y expresión } \\
\text { génica de FADS2 }\end{array}$ & $\begin{array}{l}\text { Alta concentración de D3G } \\
\text { probablemente enmascara } \\
\text { efecto de ANT sobre síntesis } \\
\text { de EPA y DHA. }\end{array}$ & $\begin{array}{l}\text { Vauzour et } \\
\text { al., } 2015^{54}\end{array}$ \\
\hline $\begin{array}{l}\text { Exp. 2: } 13 \\
\text { Muestras de plasma } \\
\text { de mujeres post } \\
\text { menopáusicas }\end{array}$ & $\begin{array}{l}\text { Exp. 2: } 12 \\
\text { semanas } \\
\text { Exp. 3: 24-48 } \\
\text { h de } \\
\text { incubación }\end{array}$ & $\begin{array}{l}\text { Exp. 2: Extracto de } \\
\text { sauco. } \\
\text { Exp. } 3 \alpha \text {-linolénico } \\
\text { (ALA), antocianinas } \\
\text { y derivados }\end{array}$ & $\begin{array}{l}\text { Exp. 2: } 500 \mathrm{mg} / \mathrm{día} \text { de } \\
\text { glicósido de cianidina, } \\
\text { a partir de extracto } \\
\text { de sauco } \\
\text { Exp. } 3: 5 \mu \mathrm{M} \\
\text { antocianinas } \\
\text { y } 50 \mu \mathrm{M} \mathrm{ALA}\end{array}$ & $\begin{array}{l}\downarrow \text { de EPA }(43 \text { y } 60 \%) \\
\text { tras } 24 \text { y } 48 \mathrm{~h} \text { de } \\
\text { incubación de } \\
\text { células HepG2, } \\
\downarrow \text { Expresión de } \\
\text { FADS2. }\end{array}$ & $\begin{array}{l}\text { Bajo nivel de estrógeno en } \\
\text { sujetos de estudio; menor } \\
\text { síntesis de EPA y DHA que } \\
\text { en hembras en edad } \\
\text { reproductiva } \\
\text { Altos niveles de ALA en la } \\
\text { dieta y cultivos celulares }\end{array}$ & \\
\hline 150 truchas arcoíris & 8 semanas & $\begin{array}{l}\text { Inclusión del 5\% } \\
\text { maíz morado(MM) } \\
\text { en la dieta) }\end{array}$ & $\begin{array}{l}\text { Concentración de } \\
\text { Peo3G, C3G y Pel3G } \\
\text { en el MM fue } \\
64.8 \pm 2.2,21.5 \pm 4.3 \\
\text { y } 18.7 \pm 1.7 \mathrm{mg} / \mathrm{g} \\
\text { respectivamente. }\end{array}$ & $\begin{array}{l}\uparrow P U F A \text { n-3 y n- } 6 \text { en } \\
\text { el tejido corporal y } \\
\text { de PUFA n-3 en el } \\
\text { plasma. } \uparrow \text { TAC en } \\
\text { plasma y expresión } \\
\text { de gpx1 en eritrocitos }\end{array}$ & $\begin{array}{l}\text { Protección in vivo contra } \\
\text { la peroxidación lipídica }\end{array}$ & $\begin{array}{l}\text { Villasante } \\
\text { et al, 2015 }\end{array}$ \\
\hline
\end{tabular}




\begin{tabular}{|c|c|c|c|c|c|c|}
\hline \begin{tabular}{|l}
48 ratas macho \\
Wistar $(75-100$ g)
\end{tabular} & 2 semanas & $\begin{array}{l}\text { Pellets con aleurona } \\
\text { de trigo (WA) y } \\
\text { control (CP). } \\
\text { Aceite de linaza }\end{array}$ & $\begin{array}{l}\text { WA y CP: } 53.4 \text { y } 8.4 \\
\text { mg/de AF/rata/día. } \\
\text { Aceite de palma (AP) y } \\
\text { aceite de linaza (AL): } \\
200 \mu \mathrm{L} / \text { día ( } 92 \mathrm{mg} / \text { día } \\
\text { de ALA). }\end{array}$ & $\begin{array}{l}\text { Polifenoles de WA } \\
\text { interfieren en } \\
\text { metabolismo de } \\
\text { AGPI CL n-3, sin } \\
\text { afectar a AGPI n-6 } \\
\text { en plasma, pero no } \\
\text { en hígado. } \\
\text { Mecanismos de } \\
\text { acción de polifenoles } \\
\text { no están totalmente } \\
\text { identificados. }\end{array}$ & $\begin{array}{l}\text { AP: Microbiota intestinal } \\
\uparrow \text { Síntesis de EPA, desde ALA. } \\
\text { AL: exceso de ALA } \downarrow \\
\text { EPA, posiblemente por inhibir } \\
\text { crecimiento de bacterias, y } \\
\text { la ruta de LCPUFA n-3 }\end{array}$ & $\begin{array}{l}\text { Ounnas } \\
\text { et al., 2014 }\end{array}$ \\
\hline $\begin{array}{l}4 \text { Vacas lecheras } \\
\text { (Holstein), } 616 \pm 53 \mathrm{~kg} \\
87 \pm 29 \text { días lactancia }\end{array}$ & $\begin{array}{l}28 \text { días } \\
\text { granada alto } \\
\text { (ECG), }\end{array}$ & $\begin{array}{l}\text { Extracto de cáscara } \\
\text { de } 1200 \mathrm{ml} \\
\text { en fenoles y taninos }\end{array}$ & $\begin{array}{l}\text { ECG: } 0,400,800 \text { y } \\
\downarrow \text { Proporción n-6/n-3 }\end{array}$ & $\begin{array}{l}\uparrow E P A \text { y DHA } \\
\text { secundarios } \\
\text { disminuyen en } \\
\text { leche. }\end{array}$ & $\begin{array}{l}\text { Posiblemente metabolitos } \\
\text { et al., } 2014^{63} \\
\text { bio-hidrogenación ruminal } \\
\text { o actividad de bacterias } \\
\text { ruminales. } \\
\uparrow \text { Biosíntesis de PUFA n-3 en } \\
\text { glándula mamaria. }\end{array}$ & Abarghuei \\
\hline $\begin{array}{l}\text { Eritrocitos de } 25 \\
\text { mujeres } \\
\text { aparentemente } \\
\text { sanas, 35.2 } 7.7 \\
\text { años }\end{array}$ & 3 meses & $\begin{array}{l}100 \mathrm{ml} \text { diarios de } \\
\text { Jugo de sauco, rico } \\
\text { en polifenoles, como } \\
\text { parte de la dieta }\end{array}$ & $\begin{array}{l}120.9 \text { mg de glucósidos } \\
\text { de antocianinas } \\
\text { y } 442 \mathrm{mg} \text { de pro- } \\
\text { antocianidinas/100 g } \\
\text { de jugo. }\end{array}$ & $\begin{array}{l}\text { Consumo de jugo de } \\
\text { sauco incrementó } \\
\text { actividad de SOD } \\
\text { y GPx } \\
\downarrow \text { la tasa AGPI n-6/n3, } \\
\uparrow \text { total AGPI n-3 }\end{array}$ & $\begin{array}{l}\text { El consumo de jugo de } \\
\text { sauco, protege indirectamente } \\
\text { a lípidos de membrana del } \\
\text { daño oxidativo, } \\
\text { al incrementar la actividad } \\
\text { enzimática de SOD y GPx }\end{array}$ & $\begin{array}{l}\text { Kardum } \\
\text { et al, } 2014^{60}\end{array}$ \\
\hline $\begin{array}{l}90 \text { ratas macho } \\
\text { Wistar (un mes de } \\
\text { edad) }\end{array}$ & 8 semanas & $\begin{array}{l}\text { Maíz genéticamente } \\
\text { modificado, rico en } \\
\text { antocianinas. }\end{array}$ & $\begin{array}{l}0.24 \pm 0.01 \mathrm{mg} \text { de } \\
\text { ANT/ gramo de pellets }\end{array}$ & $\begin{array}{l}\uparrow \text { Niveles } \\
\text { plasmáticos de } \\
\text { EPA y DHA. }\end{array}$ & $\begin{array}{l}\text { Acción de flavonoides } \\
\text { (antocianinas) sobre } \Delta 5, \\
\Delta 6 \text { D y PPAR- } \alpha \text {. }\end{array}$ & $\begin{array}{l}\text { Toufektsian } \\
\text { et al., } 2011^{13}\end{array}$ \\
\hline $\begin{array}{l}61 \text { ratas macho } \\
\text { Wistar }\end{array}$ & 7 semanas & $\begin{array}{l}\text { etanol en el agua de } \\
\text { bebida }\end{array}$ & $6 \%$ y $12 \%(v / v)$ & $\begin{array}{l}\uparrow \text { La concentración de } \\
\text { AGPI n-3 en plasma, } \\
\text { membranas celulares } \\
\text { y fosfolípidos de } \\
\text { mitocondrias } \\
\text { cardiacas. }\end{array}$ & $\begin{array}{l}\text { El estudio no fue diseñado } \\
\text { para investigar mecanismos } \\
\text { biológicos. }\end{array}$ & $\begin{array}{l}\text { Giraud et } \\
\text { al., } 2008^{48}\end{array}$ \\
\hline $\begin{array}{l}353 \text { pacientes con } \\
\text { antecedentes de } \\
\text { infarto al } \\
\text { miocardio }\end{array}$ & $\begin{array}{l}\text { Evaluación } \\
\text { del consumo } \\
\text { de ALA y } \\
\text { etanol, } \\
\text { durante } \\
27 \text { meses }\end{array}$ & $\begin{array}{l}\text { Grupo dieta } \\
\text { mediterránea rica en } \\
\text { ALA } \\
\text { Grupo control: baja } \\
\text { ingesta de ALA }\end{array}$ & $\begin{array}{l}\text { GDM: Consumo de } \\
\text { etanol: } 7.3 \% \text { de energía } \\
\text { dietaria ( } 88 \% \text { de etanol } \\
\text { del vino). } \\
\text { Grupo control: } \\
\text { consumo etanol: } 8 \% \text { de } \\
\text { la energía dietaria ( } 95 \% \\
\text { de etanol de vino) }\end{array}$ & $\begin{array}{l}\text { Alta ingesta de } \\
\text { ALA: CMV } \\
\uparrow 37 \% \text { EPA y } 10 \% \\
\text { DHA. Baja ingesta } \\
\text { de ALA, } \uparrow 50 \% \\
\text { EPA en } 50 \% \text { y } \\
21 \% \text {. de DHA } \\
\text { plasmático }\end{array}$ & $\begin{array}{l}\text { No se descarta la } \\
\text { posibilidad de que el } \\
\text { efecto sea mediado por } \\
\text { los demás componentes } \\
\text { del vino }\end{array}$ & $\begin{array}{l}\text { de Lorgeril } \\
\text { et al., } 2008^{51}\end{array}$ \\
\hline $\begin{array}{l}46 \text { hombres, de } 35 \\
\text { a } 65 \text { años de edad }\end{array}$ & 3 meses & $\begin{array}{l}\text { Bebidas alcohólicas } \\
\text { (cerveza, vino, cidra } \\
\text { aperitivos y licores) }\end{array}$ & $\begin{array}{l}0,<35 \text { y } 35-65 \text { g. de } \\
\text { alcohol/día }\end{array}$ & $\begin{array}{l}\text { Enriquecimiento de } \\
\text { AA y EPA en } \\
\text { fosfolípidos de } \\
\text { partículas de HDL }\end{array}$ & $\begin{array}{l}\text { Etanol puede ocasionar } \\
\text { cambios en la actividad } \\
\text { enzimática de } \Delta 5 \text { y } \Delta 6 \mathrm{D}\end{array}$ & $\begin{array}{l}\text { Perret et al. } \\
2002^{47}\end{array}$ \\
\hline
\end{tabular}


Empleando de jugo de sauco, pudo apreciarse el incremento del total de AGPI n-3, y de la actividad de las enzimas superóxido dismutasa (SOD) y glutatión peroxidasa (GPx) en mujeres (35.2 \pm 7.7 años), sugiriendo que los polifenoles, entre ellos antocianinas y pro-antocianidinas, protegerían indirectamente a los lípidos del daño celular, al incrementar la actividad de las enzimas antioxidantes e inhibir la formación de radicales libres, y esto redundaría en la conservación de AGPI, en particular n-360. Así mismo, se ha reportado que la inclusión de maíz morado en la dieta de truchas arcoíris, incrementa los niveles de AGPI n6 y n3 en el tejido corporal y el plasma, la capacidad antioxidante total (TAC) y la expresión de GPX-1. Por tanto, flavonoides como las antocianinas ejercerían un efecto protector contra la peroxidación lipídica ${ }^{61}$.

A favor de la hipótesis de que los flavonoides también incrementan la concentración de los AGPI mediante la modificación de la actividad enzimática o la expresión génica de las enzimas $\Delta 5$ y $\Delta 6 \mathrm{D}$, se ha informado que el consumo de una infusión de Hibiscus sabdariffa restaura la actividad de $\Delta 5$ y $\Delta 6 \mathrm{D}$ en ratas inducidas a Síndrome Metabólico, relacionando este efecto al elevado nivel de antioxidantes en la infusión ${ }^{62}$. En caso de bovinos, el consumo de un extracto de cáscara de granada, rica en polifenoles, se tradujo en mayor contenido de EPA y DHA en leche, atribuyendo este efecto a una reducción en la bio-hidrogenación ruminal y al incremento de las rutas encargadas de la síntesis de AGPI-CL en la glándula mamaria ${ }^{63}$.

Del mismo modo, la suplementación con una elevada dosis de aceite de chía como fuente de ALA inhibe la expresión génica de $\Delta 5$ y $\Delta 6 \mathrm{D}$, mientras la adición dietaria de un extracto de maíz morado, como fuente de antocianinas, incrementa la expresión de SREBP-1 y de $\Delta 5$ y $\Delta 6 \mathrm{D}$ en la glándula mamaria de ratas lactantes, probando que las estos flavonoides inciden sobre el metabolismo de los PUFA-CL ${ }^{41}$. Otros flavonoides como naringenina y hesperetina también incrementan la concentración de AGPI n-3 y reduciendo la proporción n-6/n-3, elevando adicionalmente la actividad de SOD1, SOD2, GPx, GR y GSH en el hígado de ratas macho, postulándose que estos flavonoides además de influir sobre las enzimas desaturasas $\Delta 5$ y $\Delta 6$, ejercen un control sobre el daño oxidativo ${ }^{64}$.

Además del posible efecto antioxidante y la activación de las elongaciones y desaturaciones mediadas por PPAR- $\alpha$, se ha sugerido una posible acción de los flavonoides sobre la composición de la microbiota intestinal, lo que incrementaría la síntesis de AGPI-CL n-3 al activar los procesos de elongación y desaturación, así, Ounnas ${ }^{21}$ señala que el ácido ferúlico de la aleurona de trigo incrementa la concentración de AGPI n-3 en al plasma, y que estos se relacionan con la concentración de metabolitos de polifenoles en la orina ${ }^{65}$, observando también incremento de AGPI n-3 en sangre y tejido hepático de ratas al suplementarlas con dietas que incluían $50 \%$ de centeno integral, evidenciando cambios marginales en la composición de la microbiota intestinal ${ }^{66}$, del mismo modo, al incluir hasta un $79 \%$ de trigo integral en la dieta de ratas Ounnas et al. ${ }^{67}$ informaron de modificaciones en la microbiota intestinal, e incremento de AGPI n-3 en la sangre.

\section{CONCLUSIÓN}

Los ácidos grasos alfa-linolenico (ALA, 18:3n-3) y linoleico (AL, 18:2n-6) son los precursores de los AGPI - CL n-3 y n-6, respectivamente, y son esenciales para la síntesis de los mismos, por lo que deben suministrase en la dieta.

El principal producto de AL es el ácido araquidónico, cuyos eicosanoides presentan potentes propiedades proinflamatorias, pro-agregantes y vasoconstrictoras, mientras los eicosanoides derivados de EPA, producto intermediario del metabolismo de ALA, presentan propiedades opuestas a los derivados de AGPI n-6. EI DHA, principal producto del metabolismo de ALA, genera protectinas, maresinas y resolvinas, con actividad inflamatoria.

EPA y DHA pueden obtenerse de pescados grasos o azules, desafortunadamente la sobreexplotación de estas especies y la polución de los mares restringen la disponibilidad y acceso de estos alimentos para la población.

El consumo de ALA presenta beneficios para la salud, independientemente de su síntesis hacia EPA y DHA, sin embargo, al menos parcialmente sus efectos se atribuyen a la síntesis hacia EPA y DHA.

La conversión de ALA hacia estos ácidos grasos es limitada, dependiendo de múltiples factores como la competencia de AL y ALA por las enzimas de una misma ruta metabólica, preferencia de $\triangle 6 \mathrm{D}$ por ALA, expresión génica de elongasas y desaturasas, proporción AL: ALA en la dieta, dosis de ALA, AL o de AGPI n-3 o n-6, retroalimentación negativa de la síntesis de AGPI mediada por el producto, estimulación de factores de transcripción como SREBP-1c y PPAR- $\alpha$ y factores hormonales como la insulina, que estimula la expresión de $\Delta 6 \mathrm{D}$, o el estrógeno, que al modular PPAR- $\alpha$ estimula las síntesis de PUFAS $\mathrm{n}-3$, determinando que este proceso sea más eficiente en el género femenino, además de que la conversión de AGPI es un proceso tejido-dependiente. Aún no está completamente dilucidado el efecto específico de todos estos factores y las interacciones que podrían darse entre ellos durante la síntesis de PUFAs.

En modelos animales y en humanos se ha evidenciado que la ingesta de antioxidantes contribuye a incrementar la concentración de PUFAs n-3, en este sentido se han propuesto diversos mecanismos de acción entre ellos: incremento de la actividad de las enzimas desaturasas, elongación y desaturación de ALA por la microbiota intestinal, o la incorporación de polifenoles a la bicapa de lípidos, protegiendo a DHA de la acción de radicales libres. Aún se debe corroborar si estas hipótesis son ciertas.

Agradecimientos. Sixto Reyna Gallegos es beneficiario de una beca doctoral de la Secretaría Nacional de 
Educación Superior, Ciencia, Tecnología e Innovación (SENESCYT) del gobierno ecuatoriano, por lo que deja expresa constancia de su agradecimiento a dicha institución

\section{BIBLIOGRAFÍA}

1. World Health Organization. Global status report on noncommunicable diseases 2010 [internet]; Switzerland: WHO Press; 2011 [Octubre 26, 2017] Available from: http://apps. who.int/iris/bitstream/10665/44579/1/9789240686458_eng. pdf

2. Bermúdez Ol, Toher C, Montenegro-Bethancourt C, Vossenaar $M$ et al. Dietary intakes and food sources of fat and fatty acids in Guatemalan schoolchildren: A cross-sectional study. BCM Nutrition Journal 2010; 9: 20.

3. Patterson E, Wall R, Fitzgerald DF, Ross RP et al. Health implications of high dietary omega- 6 polyunsaturated Fatty acids. J Nutr Metab 2012; 539426.

4. Flachs P, Rossmeisl M, Bryhn M, Kopecky J. Cellular and molecular effects of $n-3$ polyunsaturated fatty acids on adipose tissue biology and metabolism. Clin Sci 2009; 116: 1-16.

5. Dossi CG, Tapia GS, Espinoza A, Videla LA et al. Reversal of high-fat-diet-induced hepatic steatosis by n-3 LCPUFA: Role of PPAR- $\alpha$ and SREBP 1-c. J Nutr Biochem 2014; 25(9): 977984.

6. Lauritzen L, Brambilla P, Mazzocchi A, Harsløf LBS et al. DHA effects in brain development and function. Nutrients 2016; $8(1): 6$.

7. Iamandei G, Mocanu V, Oboroceanu T, Luca V. Involvement of N6 and N3 polyunsaturated faty acids on the lipidic profile in central nervous system of the animals of experience. Romanian Neurosurgery 2010; XVII (4): 396-402.

8. FAO. 2016. The State of World Fisheries and Aquaculture 2016 [internet] (Food and Agricultural Organization, Rome, 2016) [Octubre 28, 2017] Available from http://www.fao. org/3/a-i5555e.pdf

9. Harris WS. Alpha-linolenic: a gift from the land?. Circulation 2005; 111(22): 2872-2874.

10. Kim KB, Nam YA, Kim HS, Hayes AW et al. $\alpha$-linolenic acid: Nutraceutical, pharmacological and toxicological evaluation. Food Chem Toxicol 2014; 70: 163-178.

11. Seymour EM, Tanone II, Urcuyo-Llanes DE, Lewis SK et al. Blueberry intake alters skeletal muscle and adipose tissue peroxisome proliferator-activated receptor activity and reduces insulin resistance in obese rats. J Med Food 2011; 14(12): 1511-1518.

12. Hwang YP, Choi JH, Han EH, Kim HG et al. Purple sweet potato anthocyanins attenuate hepatic lipid accumulation through activating adenosine monophosphate-activated protein kinase in human HepG2 cells and obese mice. Nutr Res 2011; 31(12): 896-906.

13. Toufektsian MC, Salen P, Laporte F, Tonelli C et al. Dietary flavonoids increase plasma very long-chain (n-3) fatty acids in rats. J Nutr 2011; 141(1): 37-41.

14. Poudyal H, Panchal SK, Diwan V, Brown L. Omega-3 fatty acids and metabolic syndrome: Effects and emerging mechanisms of action. Prog Lipid Res 2011; 50(4): 372-387.

15. Ristic-Medic D, Vuić V, Takić M, Karadžić I et al. Polyunsaturated fatty acids in health and disease. J Serb Chem Soc 2013; 78(9): 1269-1289.

16. Barceló-Coblijin G, Murphy EJ. Alpha-linolenic acid and its conversion to longer chain n-3 fatty acids: Benefits for human health and a role in maintaining tissue $n-3$ fatty acid levels.
Prog Lipid Res 2009; 48(6): 355-374.

17. Smink W, Gerrits IW, Gloaguen M, Ruiter A et al. Linoleic and $\alpha$-linolenic acid as precursor and inhibitor for the synthesis of long-chain polyunsaturated fatty acids in liver and brain of growing pigs. Animal 2012; 6(2): 262-270.

18. Calder PC. Mechanisms of action of ( $n-3)$ fatty acids. I Nutr 2012; 142(3): 592S-599S

19. Park HG, Lawrence P, Engel MG, Kothapalli K et al. Metabolic fate of docosahexaenoic acid (DHA; 22:6n-3) in human cells: Direct retroconversion of DHA to eicosapentaenoic acid (20:5n-3) dominates over elongation to tetracosahexaenoic acid (24:6n-3). FEBS Letters 2016; 590: 3188-3194.

20. Russo GL. Dietary n-6 and n-3 polyunsaturated fatty acids: from biochemistry to clinical implications in cardiovascular prevention. Biochem Pharmacol 2009; 77(6): 937-946.

21. Ounnas $F$, Privé $F$, Salen $P$, Hazane-Puch $F$ et al. Wheat aleurone polyphenols increase plasma eicosapentaenoic acid in rats. Food Nutr Res 2014; 58: 24604.

22. Coates W. ALA Sources-Plants, Seeds, and Nuts. In F. De Meester, R. Ross and S. Zibadi, Eds. Omega-6/3 Fatty Acids. Functions, Sustainability Strategies and Perspectives. Humana Press Inc, New York, 2013, p. 309-316.

23. Matsuzaka T, Shimano $H$, Yahagi $N$, Amemiya-Kudo $M$ et al. Dual regulation of mouse $\triangle 5$ - and $\triangle 6$-desaturase gene expression by SREBP-1 and PPAR $\alpha$. I Lipid Res 2002; 43(1): 107-114.

24. Jump DB. Fatty acid regulation of hepatic lipid metabolism. Curr Opin Clin Nutr Metab Care 2011; 14 (2): 115-120.

25. Georgiadi A, Kersten S. Mechanism of gene regulation by fatty acids. Adv. Nutr 2012; 3: 127-134.

26. Shao W, Espenshade PJ. Expanding roles for SREBP in metabolism. Cell Metab 2012; 16(4): 414-419.

27. Nakamura MT, Nara TY. Essential fatty acid synthesis and its regulation in mammals. Prostaglandins Leukot Essent Fatty Acids 2003; 68(2): 145-150.

28. Ye J, DeBose-Boyd RA. Regulation of cholesterol and fatty acid synthesis. Cold Spring Harb Perspect Biol 2011; 3: a004754.

29. Dessalle K, Euthine V, Chanon S, Delarichaudy J et al. SREBP-1 Transcription factors regulate skeletal muscle cell size by controlling protein synthesis through myogenic regulatory factors. PLOS ONE 2012; 7(11): e50878.

30. Espenshade PJ. SREBPS: sterol-regulated transcription factors. J Cell Sci 2006; 119: 973-976.

31. Eberlé $D$, Hegarty $B$, Bossard P, Ferré P et al. SREBP transcription factors: master regulators of lipid homeostasis. Biochimie 2004; 86(11): 839-848.

32. Dong $X, X u H$, Mai $K, X u W$ et al. Cloning and characterization of SREBP-1 and PPAR- $\alpha$ in Japanese seabass Lateolabrax japonicus, and their gene expressions in response to different dietary fatty acids profiles. Comp Biochem Physiol B: Biochem Mol Biol 2015; 180: 48-56.

33. Barish G, Narkar V, Evans R. Review series PPAR $\delta$ : a dagger in the heart of the metabolic syndrome. J Clin Invest 2006; 116(3): 590-597.

34. Sandoval AG, Manzur F, Gómez D, Gómez C. Nuclear receptors and lipid metabolism: Cardiovascular implications. Rev. Colomb. Cardiol 2009; 16(1): 29-34.

35. Contreras AV, Torres N, Tovar AR. PPAR- $\alpha$ as a key nutritional and environmental sensor for metabolic adaptation. Adv Nutr 2013; 4(4): 439-452.

36. Fan W, Evans R. PPARs and ERRs: Molecular mediators of mitochondrial metabolism. Curr Opin Cell Biol 2015; 33: 49-54. 
37. Rodríguez-Cruz M, Tovar AR, Prado del M, Torres N. Molecular mechanisms of action and health benefits of polyunsaturated fatty acids. Rev Invest Clín 2005; 57(3): 457-472.

38. Tu WC, Cook-Johnson RJ, James MJ, Mühlhäusler BS et al. Omega-3 long chain fatty acid synthesis is regulated more by substrate levels than gene expression. Prostaglandins Leukot Essent Fatty Acids 2010; 83(2): 61-68.

39. Nara TY, He WS, Tang C, Clarke SD et al. The E-box like sterol regulatory element mediates the suppression of human Delta-6 desaturase gene by highly unsaturated fatty acids. Biochem Biophys Res Commun 2002; 296(1): 111-117.

40. Rudolph MC, McManaman IL, Phang T, Russell T, Kominsky DJ, Serkova NJ. Metabolic regulation in the lactating mammary gland: A lipid synthesizing machine. Physiol Genomics 2007; 28(3): 323-336.

41. Reyna S, Gonzáles C, Valenzuela R, Villanueva ME. Effect of purple corn extract on gene expression of srebp-1, A5d y A6d in mammary gland of nursing rats supplemented with chia oil (Salvia Hispánica L.). Rev Chil Nutr 2016; 43(3):296-302.

42. Ren T, Zhu J, Zhu L, Cheng M. The combination of blueberry juice and probiotics ameliorate non-alcoholic steatohepatitis (NASH) by affecting SREBP-1C/ PNPLA-3 pathway via PPRA- $\alpha$. Nutrients 2017; 9:198.

43. Nakamura MT, Cho HP, Clarke SD. Regulation of hepatic delta- 6 desaturase expression and its role in the polyunsaturated fatty acid inhibition of fatty acid synthase gene expression in mice. I Nutr 2000; 130(6): 1561-1565.

44. Benatti P, Peluso G, Nicolai R, Calvani M. Polyunsaturated fatty acids: Biochemical, nutritional and epigenetic properties. J Am Coll Nutr 2004; 23(4): 281-302.

45. Gibson RA, Neumann MA, Lien EL, Boyd KA et al. Docosahexaenoic acid synthesis from alpha-linolenic acid is inhibited by diets high in polyunsaturated fatty acids. Prostaglandins Leukot Essent Fatty Acids 2013; 88(1): 139146.

46. Richard IL. Coronary risk factors. The French paradox. Arch Mal Coeur Vaiss 1987; 80: 17-21.

47. Perret B, Ruidavets JB, Vieu C, Jaspard B et al. Alcohol consumption is associated with enrichment of high-density lipoprotein particles in polyunsaturated lipids and increase cholesterol esterification rate. Alcohol Clin Exp Res 2002; 26(8): 1134-1140.

48. Guiraud $A$, de Lorgeril $M$, Zeghichi S, Laporte $F$ et al. Interactions of ethanol drinking with $n-3$ fatty acids in rats: Potential consequences for cardiovascular system. Br J Nutr 100: 1237-1244.

49. Burak $C$, Wolffram $S$, Zur B. Langguth $P$, Fimmers $R$ et al. Effects of the flavonol quercetin and $\alpha$-linolenic acid on $n-3$ PUFA status in metabolically healthy men and women: $A$ randomised, double-blinded, placebo-controlled crossover trial. Br J Nutr 2017; 117(5): 698-711.

50. Di Giuseppe $R$, de Lorgeril M, Salen P, Laporte F et al. Alcohol consumption and $n-3$ polyunsaturated fatty acids in healthy men and women from 3 European populations. Am J Clin Nutr 2009; 89: 354-362.

51. de Lorgeril M, Salen P, Martin JL, Boucher F et al. Interactions of wine drinking with omega 3 fatty acids in patients with coronary heart disease: A fish-like effect of moderate wine drinking. Am Heart J 2008; 155(1): 175-181.
52. Cazzola R, Cestaro B. Red wine polyphenols protect n-3 more than $n-6$ polyunsaturated fatty acid from lipid peroxidation. Food Res Int 2011; 44: 3065-3071.

53. Graf D, Seifert S, Jaudszus A, Bub A et al. Anthocyanin rich juice lowers serum cholesterol, leptin, and resistin and improves plasma fatty acids composition in Fischer rats. PLoS ONE 2013; 8(6): e 66690.

54. Vauzour D, Tejera N, O’Neill C, Booz V et al. Anthocyanins do not influence long chain n-3 fatty acid status: studies in cells, rodents and humans. I Nutr Biochem 2015; 26: 211218.

55. Burdge GC. Wootton SA. Conversion of alpha-linolenic acid to eicosapentaenoic and docosahexaenoic acids in young women. Br J Nutr 2002; 88(4): 411-420.

56. Takikawa M, Inoue $S$, Horio F, Tsuda T. Dietary anthocyaninrich bilberry extract ameliorates hyperglycemia and insulin sensitivity via activation of AMP-activated protein kinase in diabetic mice. I Nutr 2010; 140(3): 527-533.

57. Kidson AP, Stroud CK, Stark KD. Elevated production of docosahexaenoic acid in females: Potential molecular mechanisms. Lipids 2010; 45(3) 209-224.

58. Jump DB. N-3 polyunsaturated fatty acid regulation of hepatic gene transcription. Curr Opin Lipidol 2008; 19(3): 242-247.

59. Guo H, Lui G, Zhong $R$, Wang Y et al. Cyanidin-3-O- $\beta$-glucoside regulates fatty acid metabolism via an AMP-activated protein kinase-dependent signaling pathway in human HepG2 cells. Lipids Health Dis 2012; 11: 10.

60. Kardum N. Takić M. Šavikin K. Zec M. Zdunić G. Spasić S. Konić-Ristić A. Effects of polyphenol-rich chokeberry juice on cellular antioxidant enzymes and membrane lipid status in healthy women. J Funct Foods 2014; 9: 89-97.

61. Villasante $A$, Patro B, Chew B, Becerra $M$ et al. Dietary intake of purple corn extract reduces fat body content and improves antioxidant capacity and n-3 polyunsaturated fatty acid in plasma of rainbow trout, Oncorhynchus mykiss. I World Aquacult Soc 2015; 46(4): 381-394.

62. Pérez-Torres I, Zúñiga A, Beltrán-Rodríguez U, Díaz-Díaz $E$ et al. Modification of the liver fatty acids by Hibiscus sabdariffa Linnaeus (Malvaceae) infusion, its possible effect on vascular reactivity in a metabolic syndrome model. Clin Exp Hypertens 2014; 36(3): 123-131.

63 Abarghuei MJ, Rouzbehan Y, Salem AZM, Zamiri MJ. Nitrogen balance, blood metabolites and milk fatty acid composition of dairy cows fed pomegrate - peel extract. Livest Sci 2014; 164: 72-80.

64. Miler M, Zivanović J, Ajdzanović V. Orescanin-Dusić Z et al. Citrus flavanones naringenin and hesperetin improve antioxidant status and membrane lipid compositions in the liver of old-aged Wistar rats. Exp Gerontol 2016; 84: 49-60.

65. Ounnas $F$, Salen P, Demeilliers C, Calani $L$ et al. Wheat aleurone fractions and plasma $n-3$ fatty acids in rats. Int I Food Sci Nutr 2015; 66:(4): 391-394.

66. Ounnas F, Privé $F$, Salen P, Gaci N. Whole rye consumption improves blood and liver $n-3$ fatty acid profile and gut microbiota composition in rats. PLOS ONE 2016; 11(2): e0148118.

67. Ounnas $F$, de Lorgeril $M$, Salen $P$, Laporte $F$ et al. Rye polyphenols and the metabolism of $n-3$ fatty acids in rats: a dose dependent fatty fish-like effect. Sci Rep 2017; 7: 40162. 